\title{
The Effect of Preoperative Pulmonary Arterial Hypertension on Incidence of Postoperative Atrial Fibrillation after Surgical Closure of Atrial Septal Defect (Secundum)
}

\author{
A K M MANZURULALAM, ISTIAQ AHMED, MANZIL AHMED, MOHAMMAD ASHRAF, MAMUN HOSSAIM, \\ MIZANURRAHMAN, MOHASHIN REZA \\ Department of Cardiac Surgery, National Institute of Cardiovascular Disease (NICVD) Dhaka, Bangladesh \\ Address of Correspondence: Professor AKM ManjurulAlam, Professor Cardiac Surgery, National Institute of Cardiovascular \\ Disease (NICVD) Dhaka, Bangladesh.Email:dr.manzurs@yahoo.com, mobile: 01711533223
}

\begin{abstract}
:
Bcakground: Atrial tachyarrhythmias are common among adults with ASD and contribute to substantial morbidity. Atrial flutter and atrial fibrillation are well-described complications of atrial septal defect (ASD) and are associated with substantial morbidity. The cause of AF in ASD is multifactorial in nature. It has been proposed that it is related to atrial dilatation, the increase in pulmonary pressure, and ventricular dysfunction. Early surgical intervention may reduce the long-term risk of developing atrial arrhythmias. Studies demonstrated that cardiac remodeling occurred after closure of an ASD, even in older patients. This study aimed to find out the pulmonary hypertension (PAH) as a predictor of postoperative atrial fibrillation in patients after surgery for secundum type atrial septal defect closure.

Methods: This Prospective Observational Study carried out in department of cardiac surgery, National Institute of Cardiovascular Disease (NICVD) and Hospital, Dhaka, Bangladesh during the period of July, 2015 to June, 2016. Total 54 patients are taken and then grouped into group A and group B. Each group contains 27 patients. Group A include Patients with ASD secundum without PAH. Group B Patients with ASD secundum with PAH. All patients were evaluated with M-mode, $2 D$ and color Doppler transthoracic echocardiography and ECG before operation, at discharge, after one month and at 3 months follow-up. Statistical analysis of the results was obtained by windows based computer software with statistical package for the social sciences program (SPSS version 21).

Results: To compare atrial fibrillation postoperative at discharge and postoperative after 1 and 3 months follow-up it was found In group B 8 (29.62\%) patients had AF postoperatively, during discharge 8 (29.62\%) patients had AF, after 1 month 7 (25.93\%) patient had AF, after 3 months 6 (22.22\%) patients had AF. No AF was found in Group A patients during postoperatively, during discharge, after 1 month \& 3 months follow up. Statistical significant difference was found in between two groups $(p<0.05)$.

Conclusion: The surgical correction of atrial septal defect (ASD) is safe and effective procedure. Our studyshowed that the haemodynamic and electrophysiological results of the surgical repair of ASD secundum was superior before the development of pulmonary arterial hypertension. After surgical correction of ASD secundum raised pulmonary artery pressure became lower gradually. Atrial fibrillation developed in patient group with pulmonary arterial hypertension in postoperative period remain persistent in most patients in the follow up period, few converted to sinus rhythm
\end{abstract}

Key wards: Atrial Septal defect, Atrial Fibrilation, pulmonary hypertension.

University Heart Journal 2017; 13(1): 17-20

Introduction:

Atrial septal defect is a hole of variable size in the interatrial septum. ${ }^{1}$ Atrialtachyarrhythmias, flutter and atrial fibrillation are well-documented sequelae of atrial septal defects, associated with substantial morbidity and occasionally death. ${ }^{2}$ Atrial fibrillation is a change in cardiac rhythm that results in a great increase in the mortalitymorbidity rate in adult ASD. This arrhythmia is much more frequent in patients who survive naturally into adulthood than patients who undergo surgery for ASD in childhood. ${ }^{2}$ The cause of AF in ASD has not been determined and is probably multifactorial in nature. It has been proposed that it is related to atrial dilatation, the increase in pulmonary pressure, and ventricular 
dysfunction. ${ }^{3}$ The stretching of the atrium wall prolongs the atrial refractory period heterogeneously, making the atrium more vulnerable to the induction of fibrillation. ${ }^{1}$ Remodeling of the right ventricle and the right atrium in response to long standing pressure and volume overload appears to generate the underlying arrhythmogenic substrate in patients with $\mathrm{PAH} .{ }^{2} \mathrm{~A}$ strong correlation between an increase of 40 percent or more in atrial area and the inducibility of sustained atrial flutter or fibrillation. ${ }^{4}$ Apart from the age factor, these arrhythmias may result from an increase in pulmonary artery pressures and from long-standing atrial dilatation. ${ }^{5}$ In patients with type secundum ASDs, pulmonary arterial hypertension develops slowly in response to excessive blood flow in the pulmonary circulation over a long period of time. ${ }^{3}$ Pulmonary artery systolic pressure, measured by echocardiography, as being independently correlated with mortality. A mean artery pressure mPAPe" $25 \mathrm{~mm} \mathrm{Hg}$ is considered to be diagnostic for pulmonary hypertension. ${ }^{6}$ Modulation in autonomic activity has been considered to be a factor predisposing to cardiac arrhythmias in pulmonary hypertension patients. ${ }^{6}$ Surgical closure of ASD has been shown to lower long term cardiac mortality and morbidity. Early surgical intervention may even reduce the long-term risk of developing atrial arrhythmias. Studies demonstrated that cardiac remodeling occurred after closure of an ASD, even in older patients. ${ }^{7}$ However, pulmonary pressures often remain elevated, especially in older patients. These elevated pulmonary artery pressures may cause atrial enlargement, and therefore increase the likelihood of developing late atrial arrhythmia. ${ }^{6}$ Hence the presence ofpulmonary hypertension before repair should raise awareness for the development of atrial arrhythmias after repair.

Methods: This Prospective Observational Study carried out in department of cardiac surgery, National Institute of Cardiovascular Disease (NICVD) and Hospital, Dhaka, Bangladesh during the period of July, 2015 to June, 2016 with the permission of academic council of this institute. Total 54 patients are taken and then grouped into group A and group B. Each grpup contains 27 patients. Group A include Patients with ASD secundum without PAH. Group B Patients with ASD secundum with PAH. All patients were evaluated with M-mode, 2D and color Doppler transthoracic echocardiography and ECG before operation, at discharge, after one month and at 3 months follow-up. Statistical analysis of the results was obtained by windows based computer software with statistical package for the social sciences program (SPSS version
21).

Surgical Techniques:All patients were received General Anesthesia according to standard anesthetic protocol. A uniform operative technique was used as per local institutional protocol under CPB with standard cardioplegia solution. Surgical correction of all patients included in the study was done through Standard median sternotomy and using cardiopulmonary bypass(CPB). Closure of ASD was carried out by RA tomy using pericardial patch or direct suture. The cross clamp was removed after proper elimination of air from the left heart and the patient was rewarmed and weaned from CPB. After completing operativeprocedure, Protaminesulphate (1:1 ratio) was used to reverse the heparin effect at completion of the surgical procedure. Wound was closed in layers keeping water seal drain and RV pacing wire in situ. Per operative Aortic Cross clamp time, ECCT was recorded. Per operative PAH crisis was managed accordingly to the standard protocol practiced in this institution.

\section{Results:}

The study was conducted between total 54 patients who underwent surgical closure of isolated secundum type ASD. There were 27 patients in group A and 27 patients in group B. Patients were followed up in the perioperative period, at the time of discharge, after 1 month \& 3 months of discharge. Data were collected by interview schedule and checklist, data entered into computer and data files was constructed. Data was analyzed by SPSS program tested by students ' $t$ ' test, chi square test.Mean age of the patient of group A and group B was 20.04 \pm 4.66 and $34.85 \pm 4.81$ years respectively. Statistical significant difference was found in term of age between the groups $(p<0.05)$. In $d \leq 15$ years age range there were no patient with $\mathrm{PAH}$. The number of patient with $\mathrm{PAH}$ was maximum $(62.96 \%)$ in the age group $\geq 36$ years. The male: female ratio was 1:1.5 In term of clinical complaint, In group A, $5(18.52 \%)$ had dyspnoea, $14(66.7 \%)$ had palpitation, $10(37.03 \%)$ had fatigue, 5 (18.52\%) had repeated RTI and $1(3.7 \%)$ had complain of chest pain preoperatively. In group B, 5 (18.52\%) had dyspnoea, $16(81.48 \%)$ had palpitation, 12 (43.81\%) had fatigue, 5 (18.52\%) had repeated RTI and $2(7.4 \%)$ had complain of chest pain preoperatively. Though patients with ASD secundum may remain asymptomatic. There were gradual improvement of the RA, RV dimensions and mean PA pressure from preoperative echocardiogram to follow up after 3 months of discharge after surgery.In group B total 8 patients $(29.62 \%)$ had AF postoperatively, the number was same 
during discharge, total 8 patients $(29.62 \%)$ patient had $\mathrm{AF}$, after 1 month follow up 7 patients (25.93\%) had AF, among them 1 patient was converted to sinus. After 3 months follow up total 6 patients $(22.22 \%)$ had AF. In this follow up 2 patients were converted to sinus rhythm that had previous $\mathrm{AF}$, and 1 patient newly developed $\mathrm{AF}$. No AF was found in Group A during postoperatively, during discharge, after 1 month \& 3 months follow up. Statistical significant difference was found in between two groups $(p<0.05)$. There were no statistical difference was between two groups in term of ECCT and ACTT. There were no statistical difference was between two groups in term of ICU stay \& Hospital stay. Total 5 patients had postoperative complications like bleeding, pneumonia, pneumothorax, \& wound infection without significant morbidity.

\section{Discussion:}

Ever since the establishment in 1981, National Institute of Cardio Vascular Disease (NICVD), Dhaka performing a major role in cardiac surgery. This prospective study was carried out in the department of cardiac surgery during the period of July, 2015 to June, 2016. Because NICVD play the central role in the field of cardiac surgery countrywide it is the best referral hospital for cardiac surgery. Highest number of ASD closure surgery done here in the country. The aim of this study was to determine the influence of pulmonary hypertension for atrial fibrillation $(\mathrm{AF})$ in patients undergoing surgical closure of secundum type atrial septal defect. Out of the total $(n=54)$ patients, mean age of the patient of group $A(n=27)$ and group $\mathrm{B}(\mathrm{n}=27)$ was $20.04 \pm 4.66$ and $34.85 \pm 4.81$ years respectively. Statistical significant difference was found in term of age between the groups $(p<0.05)$. In oliver et al 2002 series, mean age of the patients of group I was $22.0 \pm 7.0$ and group II was $52.0 \pm 13.0$ years which were comparable with our study. In group A, 37.03\% were male and $62.96 \%$ were female and in group B, $40.74 \%$ were male and $59.26 \%$ were female. No significant statistical difference was found in term of sex between two groups ( $p>0.05$ ). Flyer reported in 1980 , in a study that female:male ratio is $2: 1$; this ratio varies in different age group (Perloff,2012). This study was consistent with our study. In group A, 5 (18.52\%) patients had dyspnoea, $14(66.7 \%)$ patients had palpitation, $10(37.03 \%)$ patients had fatigue, 5 (18.52\%) patients had complain of chest pain preoperatively. In group B, 5 (18.52\%) patients had dyspnoea, 16 (81.48\%) patients had palpitation, 12 (43.81\%) patients had fatigue, $5(18.52 \%)$ patients had complain of chest pain preoperatively. No significant difference was observed in term of dyspnoea, palpitation, fatigue and chest pain between the groups statistically $(p>0.05)$. No significant difference was observed in term of dyspnoea, palpitation, fatigue and chest pain between the groups statistically $(p>0.05)$. This study was consistent with Kabir 1990. Preoperatively mean size of RA (44.19 \pm 2.48$) \mathrm{mm}, \mathrm{RV}(31.48 \pm 2.99) \mathrm{mm}, \mathrm{mPA}$ pressure $(22.56 \pm 1.77) \mathrm{mm} \mathrm{Hg}$, EF $(62.33 \pm 3.11) \%$ in group A and mean size of RA $(50.67 \pm 2.68) \mathrm{mm}, \mathrm{RV}$ (36.67 \pm 7.26$) \mathrm{mm}, \mathrm{mPA}$ pressure $(48.96 \pm 4.59) \mathrm{mm} \mathrm{Hg}$, EF $(61.0 \pm 3.71) \%$ in group B and at discharge mean size of RA $(37.33 \pm 2.61) \mathrm{mm}$, RV $(29.04 \pm 2.09) \mathrm{mm}, \mathrm{mPA}$ pressure $(20.74 \pm 1.89) \mathrm{mm} \mathrm{Hg}$, EF $(62.59 \pm 3.21) \%$ in group A and mean size of RA $(44.67 \pm 2.79) \mathrm{mm}, \mathrm{RV}$ $(32.52 \pm 2.19) \mathrm{mm}, \mathrm{mPA}$ pressure $(43.56 \pm 4.01) \mathrm{mm} \mathrm{Hg}$, $\mathrm{EF}(69.04 \pm 3.18) \%$ in group B. At follow up after 1 month mean size of RA $(31.70 \pm 2.32) \mathrm{mm}, \mathrm{RV}(26.81 \pm 1.78)$ $\mathrm{mm}, \mathrm{mPA}$ pressure $(19.48 \pm 1.46) \mathrm{mm} \mathrm{Hg}$, EF $(62.59 \pm 3.21) \%$ in group A and mean size of RA $(40.37 \pm 1.76) \mathrm{mm}, \mathrm{RV}(30.3 \pm 2.33) \mathrm{mm}$, mPA pressure $(37.93 \pm 4.95) \mathrm{mm} \mathrm{Hg}$, EF $(62.28 \pm 3.2) \%$ in group B. At follow up after 3 months mean size of RA (27.59 \pm 2.78$)$ $\mathrm{mm}, \mathrm{RV}(23.11 \pm 1.39) \mathrm{mm}, \mathrm{mPA}$ pressure $(18.52 \pm 1.31)$ $\mathrm{mm} \mathrm{Hg}, \mathrm{EF}(63.52 \pm 3.04) \%$ in group A and mean size of RA $(35.63 \pm 2.15) \mathrm{mm}, \mathrm{RV}(25.41 \pm 2.40) \mathrm{mm}, \mathrm{mPA}$ pressure $(31.78 \pm 5.33) \mathrm{mm} \mathrm{Hg}$, EF $(62.96 \pm 3.18) \%$ in group B. Statistical significant difference was observed in term of RA, RV dimension, mPA pressure preoperatively, at discharge, after 1 month \& 3 months between two groups $(p<0.05)$. No statistical significant difference was in EF between two groups preoperatively, at discharge, after 1 month \& 3 months ( $>0.05$ ). Statistical significant difference was observed in term of RA, RV dimension, mPA pressure preoperatively, at ischarge, after 1 month \& 3 months between two groups $(p<0.05)$. No statistical significant difference was in EF between two groups preoperatively, at discharge, after 1 month \& 3 months $(\mathrm{p}>0.05)$. This study was comparable to the study of Du et al.2001. In group B $8(29.62 \%)$ patients had AF postoperatively, during discharge 8 (29.62\%) patients had AF, after 1 month 7 (25.93\%) patient had AF, after 3 months 6 (22.22\%) patients had AF. No AF was found in Group A patients during postoperatively, during discharge, after 1 month \& 3 months follow up. Statistical significant difference was found in between two groups $(\mathrm{p}<0.05)$. This study was consistent with de Bruaene 2010. Mean extracorporeal circulation time (ECCT) and Aortic cross clamp time (ACCT) in group A was 54.67 0.68 minutes and $29.96 \pm 0.94$ minutes respectively \& Mean extracorporeal 
circulation time (ECCT) and Aortic cross clamp time in group B was $55.19 \pm 1.3$ minutes and $30.44 \pm 1.58$ minutes respectively. No statistical difference was between two groups $(\mathrm{p}>0.05)$. Mean cardiopulmonary bypass time in Gatzoulis et al 1999 series was $42.8 \pm 24$ minutes and mean ACCT was $25.2 \pm 17$ minutes which is consistent with our study. Mean ICU stay and hospital stay was $3.30 \pm 0.47$ and $11.59 \pm 1.58$ respectively in group A \& in group B Mean ICU stay and hospital stay was $4.48 \pm 5.7$ and $11.85 \pm 1.59$ respectively. No statistical difference was between two groups $(p>0.05)$. This study was comparable to the study of Doll et al, 2001. During the postoperative period post-operative bleeding was in 1 $(3.70 \%)$ in both groups. There was post-operative pneumonia in $1(3.70 \%)$, pneumothorax in $1(3.70 \%)$, wound infection was in $1(3.70 \%)$ in group B. No statistical difference was between two groups ( $\mathrm{p}>0.05)$. All the patients recover well. No readmission required.

\section{Conclusion:}

The surgical correction of atrial septal defect (ASD) is safe and effective procedure. Our study showed that the haemodynamic and electrophysiological results of the surgical repair of ASD secundum was superior before the development of pulmonary arterial hypertension. Heart chamber especially right chambers were found dilated and dilated chambers were reduced gradually in size subsequently, seen during follow up periods in both groups. After surgical correction of ASD secundum raised pulmonary artery pressure became lower gradually within study period. Atrial fibrillation developed in patient group with pulmonary arterial hypertension in postoperative period remain persistent in most patients in the follow up period, few converted to sinus rhythm. From above findings we should concentrate our efforts on trying to close all ASD secundum before the development of PAH \& intimate follow up the patients who underwent surgical closure of ASD had PAH preoperatively to reduce the morbidity and mortality related to atrial fibrillation.

\section{Referrance:}

1. Berger F, Vogel M, Kramer A, et al. Incidence of atrial flutter/ fibrillation in adults with atrial septal defect before and after surgery. Ann Thorac Surg. 1999;68(1):75-78. doi:10.1016/S00034975(99)00478-6

2. Gatzoulis MA, Freeman MA, Siu SC, Webb GD, Harris L. Atrial Arrhythmia after Surgical Closure of Atrial Septal Defects in Adults. $N$ Engl J Med. 1999;340(11):839-846. doi:10.1056/ NEJM199903183401103

3. Toyono M. Pulmonary arterial hypertension in adults with atrial septal defect. J Cardiol Cases. 2012;6(1):e32-e33. doi:10.1016/ j.jccase.2012.04.005

4. Attie F, Rosas M, Granados N, Zabal C, Buendía A, Calderón J. Surgical treatment for secundum atrial septal defects in patients $>40$ years old: A randomized clinical trial. $\mathrm{J} \mathrm{Am} \mathrm{Coll} \mathrm{Cardiol.}$ 2001;38(7):2035-2042. doi:10.1016/S0735-1097(01)01635-7

5. Oliver JM, Gallego P, González AE, et al. [Surgical closure of atrial septal defect before or after the age of 25 years. Comparison with the natural history of unoperated patients]. Rev española Cardiol. 2002;55(9):953-961. http://www.ncbi.nlm.nih.gov/pubmed/12236925.

6. Post MC. Association between pulmonary hypertension and an atrial septal defect. Netherlands Hear J. 2013;21(7-8):331-32. doi:10.1007/s12471-013-0432-9

7. Lam CSP, Borlaug BA, Kane GC, Enders FT, Rodeheffer RJ, Redfield MM. Age-associated increases in pulmonary artery systolic pressure in the general population. Circulation. 2009;119(20):266370. doi:10.1161/CIRCULATIONAHA. 108.838698 